\title{
Approximation by complex Durrmeyer-Stancu type operators in compact disks
}

\author{
Mei-Ying Ren ${ }^{1 *}$, Xiao-Ming Zeng $^{2}$ and Liang Zeng ${ }^{2}$
}

\section{"Correspondence:}

npmeiyingr@163.com

'Department of Mathematics and

Computer Science, Wuyi University,

Wuyishan, 354300, China

Full list of author information is

available at the end of the article

\begin{abstract}
In this paper we introduce a class of complex Stancu-type Durrmeyer operators and study the approximation properties of these operators. We obtain a

Voronovskaja-type result with quantitative estimate for these operators attached to analytic functions on compact disks. We also study the exact order of approximation. More important, our results show the overconvergence phenomenon for these complex operators.
\end{abstract}

MSC: $30 \mathrm{E} 10 ; 41 \mathrm{~A} 25 ; 41 \mathrm{~A} 36$

Keywords: complex Durrmeyer-Stancu type operators; Voronovskaja-type result; exact order of approximation; simultaneous approximation; overconvergence

\section{Introduction}

In 1986, some approximation properties of complex Bernstein polynomials in compact disks were initially studied by Lorentz [1]. Very recently, the problem of the approximation of complex operators has been causing great concern, which has become a hot topic of research. A Voronovskaja-type result with quantitative estimate for complex Bernstein polynomials in compact disks was obtained by Gal [2] Also, in [3-18] similar results for complex Bernstein-Kantorovich polynomials, Bernstein-Stancu polynomials, KantorovichSchurer polynomials, Kantorovich-Stancu polynomials, complex Favard-Szász-Mirakjan operators, complex Beta operators of first kind, complex Baskajov-Stancu operators, complex Bernstein-Durrmeyer polynomials, complex genuine Durrmeyer-Stancu polynomials and complex Bernstein-Durrmeyer operators based on Jacobi weights were obtained.

The aim of the present article is to obtain approximation results for complex DurrmeyerStancu type operators which are defined for $f:[0,1] \rightarrow \mathbf{C}$ continuous on $[0,1]$ by

$$
\begin{aligned}
M_{n}^{(\alpha, \beta)}(f ; z):= & n \sum_{k=1}^{n} p_{n, k}(z) \int_{0}^{1} p_{n-1, k-1}(t) f\left(\frac{n t+\alpha}{n+\beta}\right) d t \\
& +f\left(\frac{\alpha}{n+\beta}\right) p_{n, 0}(z),
\end{aligned}
$$

where $\alpha, \beta$ are two given real parameters satisfying the condition $0 \leq \alpha \leq \beta, z \in \mathbf{C}, n=$ $1,2, \ldots$, and $p_{n, k}(z)=\left(\begin{array}{l}n \\ k\end{array}\right) z^{k}(1-z)^{n-k}$.

(c) 2013 Ren et al.; licensee Springer. This is an Open Access article distributed under the terms of the Creative Commons Attribution License (http://creativecommons.org/licenses/by/2.0), which permits unrestricted use, distribution, and reproduction in any medium, provided the original work is properly cited. 
Note that, for $\alpha=\beta=0$, these operators become the complex Durrmeyer-type operators $M_{n}(f ; z)=M_{n}^{(0,0)}(f ; z)$, this case has been investigated in [11].

\section{Auxiliary results}

In the sequel, we shall need the following auxiliary results.

Lemma 1 Let $e_{m}(z)=z^{m}, m \in \mathbf{N} \cup\{0\}, z \in \mathbf{C}, n \in \mathbf{N}, 0 \leq \alpha \leq \beta$, then we have that $M_{n}^{(\alpha, \beta)}\left(e_{m} ; z\right)$ is a polynomial of degree less than or equal to $\min (m, n)$ and

$$
M_{n}^{(\alpha, \beta)}\left(e_{m} ; z\right)=\sum_{j=0}^{m}\left(\begin{array}{c}
m \\
j
\end{array}\right) \frac{n^{j} \alpha^{m-j}}{(n+\beta)^{m}} M_{n}\left(e_{j} ; z\right) .
$$

Proof By the definition given by (1), the proof is easy, here the proof is omitted.

Let $m=0,1,2$, according to [11, Lemma 1], by a simple computation, we have

$$
\begin{aligned}
M_{n}^{(\alpha, \beta)}\left(e_{0} ; z\right)= & 1 \\
M_{n}^{(\alpha, \beta)}\left(e_{1} ; z\right)= & \frac{n^{2} z}{(n+1)(n+\beta)}+\frac{\alpha}{n+\beta} ; \\
M_{n}^{(\alpha, \beta)}\left(e_{2} ; z\right)= & \frac{n^{2}}{(n+\beta)^{2}}\left[\frac{2 n z+n(n-1) z^{2}}{(n+1)(n+2)}\right] \\
& +\frac{2 n^{2} \alpha z}{(n+1)(n+\beta)^{2}}+\frac{\alpha^{2}}{(n+\beta)^{2}} .
\end{aligned}
$$

Lemma 2 Let $e_{m}(z)=z^{m}, m \in \mathbf{N} \cup\{0\}, z \in \mathbf{C}, n \in \mathbf{N}, 0 \leq \alpha \leq \beta$, for all $|z| \leq r, r \geq 1$, we have $\left|M_{n}^{(\alpha, \beta)}\left(e_{m} ; z\right)\right| \leq r^{m}$.

Proof The proof follows directly Lemma 1 and [11, Lemma 2].

Lemma 3 Let $e_{m}(z)=z^{m}, m, n \in \mathbf{N}, z \in \mathbf{C}$ and $0 \leq \alpha \leq \beta$, then we have

$$
\begin{aligned}
M_{n}^{(\alpha, \beta)}\left(e_{m+1} ; z\right)= & \frac{z(1-z) n}{(n+\beta)(m+n+1)}\left(M_{n}^{(\alpha, \beta)}\left(e_{m} ; z\right)\right)^{\prime} \\
& +\frac{(m+n z) n+\alpha(1+2 m+n)}{(n+\beta)(m+n+1)} M_{n}^{(\alpha, \beta)}\left(e_{m} ; z\right) \\
& -\frac{\alpha m(n+\alpha)}{(n+\beta)^{2}(m+n+1)} M_{n}^{(\alpha, \beta)}\left(e_{m-1} ; z\right) .
\end{aligned}
$$

Proof Let

$$
\begin{aligned}
& T_{n-1, k-1}^{(\alpha, \beta)}(f):=\int_{0}^{1} p_{n-1, k-1}(t) f\left(\frac{n t+\alpha}{n+\beta}\right) d t, \\
& \widetilde{T}_{n-1, k-1}^{(\alpha, \beta)}(f):=\int_{0}^{1} p_{n-1, k-1}(t) t f\left(\frac{n t+\alpha}{n+\beta}\right) d t, \\
& \widehat{T}_{n-1, k-1}^{(\alpha, \beta)}(f):=\int_{0}^{1} p_{n-1, k-1}(t) t^{2} f\left(\frac{n t+\alpha}{n+\beta}\right) d t, \\
& E_{n}^{(\alpha, \beta)}(f ; z):=n \sum_{k=1}^{n} p_{n, k}(z) T_{n-1, k-1}^{(\alpha, \beta)}(f),
\end{aligned}
$$


then we have

$$
\begin{aligned}
M_{n}^{(\alpha, \beta)}(f ; z)= & E_{n}^{(\alpha, \beta)}(f ; z)+f\left(\frac{\alpha}{n+\beta}\right) p_{n, 0}(z), \\
\widetilde{T}_{n-1, k-1}^{(\alpha, \beta)}\left(e_{m}\right)= & \int_{0}^{1} p_{n-1, k-1}(t) \frac{n+\beta}{n}\left(\frac{n t+\alpha}{n+\beta}-\frac{\alpha}{n+\beta}\right)\left(\frac{n t+\alpha}{n+\beta}\right)^{m} d t \\
= & \frac{n+\beta}{n} T_{n-1, k-1}^{(\alpha, \beta)}\left(e_{m+1}\right)-\frac{\alpha}{n} T_{n-1, k-1}^{(\alpha, \beta)}\left(e_{m}\right), \\
\widehat{T}_{n-1, k-1}^{(\alpha, \beta)}\left(e_{m}\right)= & \int_{0}^{1} p_{n-1, k-1}(t)\left(\frac{n+\beta}{n}\right)^{2}\left(\frac{n t+\alpha}{n+\beta}-\frac{\alpha}{n+\beta}\right)^{2}\left(\frac{n t+\alpha}{n+\beta}\right)^{m} d t \\
= & \left(\frac{n+\beta}{n}\right)^{2} T_{n-1, k-1}^{(\alpha, \beta)}\left(e_{m+2}\right)-\frac{2 \alpha(n+\beta)}{n^{2}} T_{n-1, k-1}^{(\alpha, \beta)}\left(e_{m+1}\right) \\
& +\left(\frac{\alpha}{n}\right)^{2} T_{n-1, k-1}^{(\alpha, \beta)}\left(e_{m}\right) .
\end{aligned}
$$

By a simple calculation, we obtain

$$
z(1-z) p_{n, k}^{\prime}(z)=(k-n z) p_{n, k}(z), \quad[(k-1)-(n-1) t] p_{n-1, k-1}(t)=t(1-t) p_{n-1, k-1}^{\prime}(t)
$$

It follows that

$$
\begin{aligned}
z(1-z)\left(E_{n}^{(\alpha, \beta)}\left(e_{m} ; z\right)\right)^{\prime} & n \sum_{k=1}^{n}(k-n z) p_{n, k}(z) \int_{0}^{1} p_{n-1, k-1}(t)\left(\frac{n t+\alpha}{n+\beta}\right)^{m} d t \\
= & n \sum_{k=1}^{n} p_{n, k}(z) \int_{0}^{1}[(k-1)-(n-1) t+(n-1) t+1] p_{n-1, k-1}(t) \\
& \cdot\left(\frac{n t+\alpha}{n+\beta}\right)^{m} d t-n z E_{n}^{(\alpha, \beta)}\left(e_{m} ; z\right),
\end{aligned}
$$

where

$$
\begin{aligned}
& n \sum_{k=1}^{n} p_{n, k}(z) \int_{0}^{1}[(k-1)-(n-1) t+(n-1) t+1] \\
& \quad \cdot p_{n-1, k-1}(t)\left(\frac{n t+\alpha}{n+\beta}\right)^{m} d t \\
& =n \sum_{k=1}^{n} p_{n, k}(z) \int_{0}^{1} t(1-t) p_{n-1, k-1}^{\prime}(t)\left(\frac{n t+\alpha}{n+\beta}\right)^{m} d t \\
& \quad+n(n-1) \sum_{k=1}^{n} p_{n, k}(z) \widetilde{T}_{n-1, k-1}^{(\alpha, \beta)}\left(e_{m}\right)+n \sum_{k=1}^{n} p_{n, k}(z) T_{n-1, k-1}^{(\alpha, \beta)}\left(e_{m}\right) \\
& =n \sum_{k=1}^{n} p_{n, k}(z) \int_{0}^{1} t(1-t) p_{n-1, k-1}^{\prime}(t)\left(\frac{n t+\alpha}{n+\beta}\right)^{m} d t \\
& \quad+\frac{(n-1)(n+\beta)}{n} E_{n}^{(\alpha, \beta)}\left(e_{m+1} ; z\right)+\left[1-\frac{\alpha(n-1)}{n}\right] E_{n}^{(\alpha, \beta)}\left(e_{m} ; z\right) .
\end{aligned}
$$


Also, using integration by parts, we have

$$
\begin{aligned}
\int_{0}^{1} t & (1-t) p_{n-1, k-1}^{\prime}(t)\left(\frac{n t+\alpha}{n+\beta}\right)^{m} d t \\
= & -\int_{0}^{1} p_{n-1, k-1}(t)(1-2 t)\left(\frac{n t+\alpha}{n+\beta}\right)^{m} d t \\
& \quad-\frac{m n}{n+\beta} \int_{0}^{1} p_{n-1, k-1}(t) t(1-t)\left(\frac{n t+\alpha}{n+\beta}\right)^{m-1} d t \\
= & -T_{n-1, k-1}^{(\alpha, \beta)}\left(e_{m}\right)+2 \widetilde{T}_{n-1, k-1}^{(\alpha, \beta)}\left(e_{m}\right) \\
& -\frac{m n}{n+\beta} \widetilde{T}_{n-1, k-1}^{(\alpha, \beta)}\left(e_{m-1}\right)+\frac{m n}{n+\beta} \widehat{T}_{n-1, k-1}^{(\alpha, \beta)}\left(e_{m-1}\right) \\
= & \frac{n+\beta}{n}(m+2) T_{n-1, k-1}^{(\alpha, \beta)}\left(e_{m+1}\right)-\left(1+\frac{2 \alpha}{n}+m+\frac{2 \alpha m}{n}\right) T_{n-1, k-1}^{(\alpha, \beta)}\left(e_{m}\right) \\
& +\frac{\alpha m(\alpha+n)}{n(n+\beta)} T_{n-1, k-1}^{(\alpha, \beta)}\left(e_{m-1}\right) .
\end{aligned}
$$

So, in conclusion, we have

$$
\begin{aligned}
z(1-z)\left(E_{n}^{(\alpha, \beta)}\left(e_{m} ; z\right)\right)^{\prime}= & \frac{n+\beta}{n}(m+n+1) E_{n}^{(\alpha, \beta)}\left(e_{m+1} ; z\right) \\
& -\left[\frac{\alpha(1+2 m+n)}{n}+m+n z\right] E_{n}^{(\alpha, \beta)}\left(e_{m} ; z\right) \\
& +\frac{\alpha m(n+\alpha)}{n(n+\beta)} E_{n}^{(\alpha, \beta)}\left(e_{m-1} ; z\right),
\end{aligned}
$$

which implies the recurrence in the statement.

Lemma 4 Let $n \in \mathbf{N}, m=2,3, \ldots, e_{m}(z)=z^{m}, S_{n, m}^{(\alpha, \beta)}(z):=M_{n}^{(\alpha, \beta)}\left(e_{m} ; z\right)-z^{m}, z \in \mathbf{C}$ and $0 \leq$ $\alpha \leq \beta$, we have

$$
\begin{aligned}
S_{n, m}^{(\alpha, \beta)}(z)= & \frac{z(1-z) n}{(n+\beta)(m+n)}\left(M_{n}^{(\alpha, \beta)}\left(e_{m-1} ; z\right)\right)^{\prime} \\
& +\frac{(m-1+n z) n+\alpha(m-1+n)}{(n+\beta)(m+n)} S_{n, m-1}^{(\alpha, \beta)}(z) \\
& +\frac{\alpha m}{(n+\beta)(m+n)} M_{n}^{(\alpha, \beta)}\left(e_{m-1} ; z\right) \\
& -\frac{\alpha(m-1)(n+\alpha)}{(n+\beta)^{2}(m+n)} M_{n}^{(\alpha, \beta)}\left(e_{m-2} ; z\right) \\
& +\frac{(m-1+n z) n+\alpha(m-1+n)}{(n+\beta)(m+n)} z^{m-1}-z^{m} .
\end{aligned}
$$

Proof Using the recurrence formula (2), by a simple calculation, we can easily get the recurrence (3), the proof is omitted.

\section{Main results}

The first main result is expressed by the following upper estimates. 
Theorem 1 Let $0 \leq \alpha \leq \beta, 1 \leq r \leq R, D_{R}=\{z \in \mathbf{C}:|z|<R\}$. Suppose that $f: D_{R} \rightarrow \mathbf{C}$ is analytic in $D_{R}$, i.e., $f(z)=\sum_{m=0}^{\infty} c_{m} z^{m}$ for all $z \in D_{R}$.

(i) For all $|z| \leq r$ and $n \in \mathbf{N}$, we have

$$
\left|M_{n}^{(\alpha, \beta)}(f ; z)-f(z)\right| \leq \frac{K_{r}^{(\alpha, \beta)}(f)}{n}
$$

where $K_{r}^{(\alpha, \beta)}(f)=(1+r) \sum_{m=1}^{\infty}\left|c_{m}\right| m(m+1+\alpha+\beta) r^{m-1}<\infty$.

(ii) (Simultaneous approximation) If $1 \leq r<r_{1}<R$ are arbitrarily fixed, then for all $|z| \leq r$ and $n, p \in \mathbf{N}$, we have

$$
\left|\left(M_{n}^{(\alpha, \beta)}(f ; z)\right)^{(p)}-f^{(p)}(z)\right| \leq \frac{K_{r_{1}}^{(\alpha, \beta)}(f) p ! r_{1}}{(n+\beta)\left(r_{1}-r\right)^{p+1}}
$$

where $K_{r_{1}}^{(\alpha, \beta)}(f)$ is defined as in the above point (i).

Proof Taking $e_{m}(z)=z^{m}$, by the hypothesis that $f(z)$ is analytic in $D_{R}$, i.e., $f(z)=\sum_{m=0}^{\infty} c_{m} z^{m}$ for all $z \in D_{R}$, it is easy for us to obtain

$$
M_{n}^{(\alpha, \beta)}(f ; z)=\sum_{m=0}^{\infty} c_{m} M_{n}^{(\alpha, \beta)}\left(e_{m} ; z\right)
$$

Therefore, we get

$$
\begin{aligned}
\left|M_{n}^{(\alpha, \beta)}(f ; z)-f(z)\right| & \leq \sum_{m=0}^{\infty}\left|c_{m}\right| \cdot\left|M_{n}^{(\alpha, \beta)}\left(e_{m} ; z\right)-e_{m}(z)\right| \\
& =\sum_{m=1}^{\infty}\left|c_{m}\right| \cdot\left|M_{n}^{(\alpha, \beta)}\left(e_{m} ; z\right)-e_{m}(z)\right|,
\end{aligned}
$$

as $M_{n}^{(\alpha, \beta)}\left(e_{0} ; z\right)=e_{0}(z)=1$.

(i) For $m \in \mathbf{N}$, taking into account that $M_{n}^{(\alpha, \beta)}\left(e_{m-1} ; z\right)$ is a polynomial of degree $\leq \min (m-$ $1, n)$, by the well-known Bernstein inequality and Lemma 2 , we get

$$
\left|\left(M_{n}^{(\alpha, \beta)}\left(e_{m-1} ; z\right)\right)^{\prime}\right| \leq \frac{m-1}{r} \max \left\{\left|M_{n}^{(\alpha, \beta)}\left(e_{m-1} ; z\right)\right|:|z| \leq r\right\} \leq(m-1) r^{m-2} .
$$

On the one hand, when $m=1$, for $|z| \leq r$, by Lemma 1, we have

$$
\left|M_{n}^{(\alpha, \beta)}\left(e_{1} ; z\right)-e_{1}(z)\right|=\left|\frac{n^{2} z}{(n+1)(n+\beta)}+\frac{\alpha}{n+\beta}-z\right| \leq \frac{1+r}{n}(2+\alpha+\beta) .
$$

When $m \geq 2$, for $n \in \mathbf{N},|z| \leq r, 0 \leq \alpha \leq \beta$, in view of $|(m-1+n z) n+\alpha(m-1+n)| \leq$ $(n+\beta)(m+n) r$, using the recurrence formula (3) and the above inequality, we have

$$
\begin{aligned}
\left|M_{n}^{(\alpha, \beta)}\left(e_{m} ; z\right)-e_{m}(z)\right| & =\left|S_{n, m}^{(\alpha, \beta)}(z)\right| \\
& \leq \frac{r(1+r)}{n} \cdot(m-1) r^{m-2}+r\left|S_{n, m-1}^{(\alpha, \beta)}(z)\right|
\end{aligned}
$$




$$
\begin{aligned}
& +\frac{\alpha}{n} r^{m-1}+\frac{\alpha}{n} r^{m-2}+\frac{m+1+\beta}{n}(1+r) r^{m-1} \\
\leq & \frac{m-1}{n}(1+r) r^{m-1}+r\left|S_{n, m-1}^{(\alpha, \beta)}(z)\right| \\
& +\frac{\alpha}{n}(1+r) r^{m-1}+\frac{m+1+\beta}{n}(1+r) r^{m-1} \\
= & r\left|S_{n, m-1}^{(\alpha, \beta)}(z)\right|+\frac{2 m+\alpha+\beta}{n}(1+r) r^{m-1} .
\end{aligned}
$$

By writing the last inequality, for $m=2, \ldots$, we easily obtain step by step the following:

$$
\begin{aligned}
& \left|M_{n}^{(\alpha, \beta)}\left(e_{m} ; z\right)-e_{m}(z)\right| \\
& \leq r\left(r\left|S_{n, m-2}^{(\alpha, \beta)}(z)\right|+\frac{2(m-1)+\alpha+\beta}{n}(1+r) r^{m-2}\right) \\
& \quad+\frac{2 m+\alpha+\beta}{n}(1+r) r^{m-1} \\
& \quad r^{2}\left|S_{n, m-2}^{(\alpha, \beta)}(z)\right|+\frac{2(m-1+m)+2(\alpha+\beta)}{n}(1+r) r^{m-1} \\
& \leq \cdots \leq \frac{1+r}{n} m(m+1+\alpha+\beta) r^{m-1}
\end{aligned}
$$

In conclusion, for any $m, n \in \mathbf{N},|z| \leq r, 0 \leq \alpha \leq \beta$, we have

$$
\left|M_{n}^{(\alpha, \beta)}\left(e_{m} ; z\right)-e_{m}(z)\right| \leq \frac{1+r}{n} m(m+1+\alpha+\beta) r^{m-1}
$$

from which it follows that

$$
\left|M_{n}^{(\alpha, \beta)}(f ; z)-f(z)\right| \leq \frac{1+r}{n} \sum_{m=1}^{\infty}\left|c_{m}\right| m(m+1+\alpha+\beta) r^{m-1}
$$

By assuming that $f(z)$ is analytic in $D_{R}$, we have $f^{(2)}(z)=\sum_{m=2}^{\infty} c_{m} m(m-1) z^{m-2}$ and the series is absolutely convergent in $|z| \leq r$, so we get $\sum_{m=2}^{\infty}\left|c_{m}\right| m(m-1) r^{m-2}<\infty$, which implies $K_{r}^{(\alpha, \beta)}(f)=(1+r) \sum_{m=1}^{\infty}\left|c_{m}\right| m(m+1+\alpha+\beta) r^{m-1}<\infty$.

(ii) For the simultaneous approximation, denoting by $\Gamma$ the circle of radius $r_{1}>r$ and center 0 , since for any $|z| \leq r$ and $v \in \Gamma$, we have $|v-z| \geq r_{1}-r$. By Cauchy's formula, it follows that for all $|z| \leq r$ and $n \in \mathbf{N}$, we have

$$
\begin{aligned}
\left|\left(M_{n}^{(\alpha, \beta)}(f ; z)\right)^{(p)}-f^{(p)}(z)\right| & =\frac{p !}{2 \pi}\left|\int_{\Gamma} \frac{M_{n}^{(\alpha, \beta)}(f ; v)-f(v)}{(v-z)^{p+1}} d v\right| \\
& \leq \frac{K_{r_{1}}^{(\alpha, \beta)}(f)}{n} \frac{p !}{2 \pi} \frac{2 \pi r_{1}}{\left(r_{1}-r\right)^{p+1}} \\
& =\frac{K_{r_{1}}^{(\alpha, \beta)}(f)}{n} \cdot \frac{p ! r_{1}}{\left(r_{1}-r\right)^{p+1}}
\end{aligned}
$$

which proves the theorem.

Theorem 2 Let $0 \leq \alpha \leq \beta, R>1, D_{R}=\{z \in \mathbf{C}:|z|<R\}$. Suppose that $f: D_{R} \rightarrow \mathbf{C}$ is analytic in $D_{R}$, i.e., $f(z)=\sum_{k=0}^{\infty} c_{k} z^{k}$ for all $z \in D_{R}$. For any fixed $r \in[1, R]$ and all $n \in \mathbf{N},|z| \leq r$, we 
have

$$
\begin{aligned}
& \left|M_{n}^{(\alpha, \beta)}(f ; z)-f(z)-\frac{\alpha-(1+\beta) z}{n} f^{\prime}(z)-\frac{z(1-z)}{n} f^{\prime \prime}(z)\right| \\
& \quad \leq \frac{M_{r}(f)}{n^{2}}+\frac{M_{r, 1}^{(\alpha, \beta)}(f)}{n(n+\beta)}+\frac{M_{r, 2}^{(\alpha, \beta)}(f)}{(n+\beta)^{2}}
\end{aligned}
$$

where $M_{r}(f)=\sum_{k=1}^{\infty}\left|c_{k}\right| k B_{k, r} r^{k}<\infty$ with $B_{k, r}=r^{2}\left(2 k^{3}+3 k^{2}+3 k+1\right)+r\left(4 k^{3}+12 k^{2}+14 k+\right.$ $6)+\left(2 k^{3}+9 k^{2}+13 k+6\right), M_{r, 1}^{(\alpha, \beta)}(f)=\sum_{k=1}^{\infty}\left|c_{k}\right|\left[2 k(k-1)^{2} \alpha+2 k^{3} \beta r+k^{2} \alpha \beta+k^{2} \beta^{2} r\right] r^{k-1}$, $M_{r, 2}^{(\alpha, \beta)}(f)=\sum_{k=1}^{\infty}\left|c_{k}\right| \frac{k(k-1)\left(\alpha^{2}+\beta^{2} r^{2}\right)}{2} r^{k-2}<\infty$.

Proof For all $z \in D_{R}$, we have

$$
\begin{aligned}
& M_{n}^{(\alpha, \beta)}(f ; z)-f(z)-\frac{\alpha-(1+\beta) z}{n} f^{\prime}(z)-\frac{z(1-z)}{n} f^{\prime \prime}(z) \\
& =M_{n}^{(\alpha, \beta)}(f ; z)-f(z)+\frac{z}{n} f^{\prime}(z)-\frac{z(1-z)}{n} f^{\prime \prime}(z)-\frac{\alpha-\beta z}{n} f^{\prime}(z) \\
& =\quad\left[M_{n}(f ; z)-f(z)-\frac{z(1-z) f^{\prime \prime}(z)-z f^{\prime}(z)}{n}\right] \\
& \quad+\left[M_{n}^{(\alpha, \beta)}(f ; z)-M_{n}(f ; z)-\frac{\alpha-\beta z}{n} f^{\prime}(z)\right] \\
& :=I_{1}+I_{2} .
\end{aligned}
$$

By [11, Theorem 1], we have $\left|I_{1}\right| \leq \frac{M_{r}(f)}{n^{2}}$, where $M_{r}(f)=\sum_{k=1}^{\infty}\left|c_{k}\right| k B_{k, r} r^{k}<\infty$ with $B_{k, r}=$ $r^{2}\left(2 k^{3}+3 k^{2}+3 k+1\right)+r\left(4 k^{3}+12 k^{2}+14 k+6\right)+\left(2 k^{3}+9 k^{2}+13 k+6\right)$.

Next, let us estimate $\left|I_{2}\right|$.

By $f$ is analytic in $D_{R}$, i.e., $f(z)=\sum_{k=0}^{\infty} c_{k} z^{k}$ for all $z \in D_{R}$, we have

$$
\begin{aligned}
\left|I_{2}\right| & =\left|\sum_{k=1}^{\infty} c_{k}\left[M_{n}^{(\alpha, \beta)}\left(e_{k} ; z\right)-M_{n}\left(e_{k} ; z\right)-\frac{\alpha-\beta z}{n} k z^{k-1}\right]\right| \\
& \leq \sum_{k=1}^{\infty}\left|c_{k}\right|\left|M_{n}^{(\alpha, \beta)}\left(e_{k} ; z\right)-M_{n}\left(e_{k} ; z\right)-\frac{\alpha-\beta z}{n} k z^{k-1}\right| .
\end{aligned}
$$

On the one hand, when $k \geq 2$, since $\frac{n^{k}}{(n+\beta)^{k}}-1=-\sum_{j=0}^{k-1}\left(\begin{array}{l}k \\ j\end{array}\right) \frac{n^{j} \beta^{k-j}}{(n+\beta)^{k}}$, by Lemma 1, we obtain

$$
\begin{aligned}
& M_{n}^{(\alpha, \beta)}\left(e_{k} ; z\right)-M_{n}\left(e_{k} ; z\right)-\frac{\alpha-\beta z}{n} k z^{k-1} \\
& =\sum_{j=0}^{k-1}\left(\begin{array}{c}
k \\
j
\end{array}\right) \frac{n^{j} \alpha^{k-j}}{(n+\beta)^{k}} M_{n}\left(e_{j} ; z\right)+\left[\frac{n^{k}}{(n+\beta)^{k}}-1\right] M_{n}\left(e_{k} ; z\right) \\
& \quad-\frac{\alpha-\beta z}{n} k z^{k-1} \\
& =\sum_{j=0}^{k-2}\left(\begin{array}{c}
k \\
j
\end{array}\right) \frac{n^{j} \alpha^{k-j}}{(n+\beta)^{k}} M_{n}\left(e_{j} ; z\right)+\frac{k n^{k-1} \alpha}{(n+\beta)^{k}} M_{n}\left(e_{k-1} ; z\right)
\end{aligned}
$$




$$
\begin{aligned}
& -\sum_{j=0}^{k-1}\left(\begin{array}{l}
k \\
j
\end{array}\right) \frac{n^{j} \beta^{k-j}}{(n+\beta)^{k}} M_{n}\left(e_{k} ; z\right)-\frac{\alpha-\beta z}{n} k z^{k-1} \\
= & \sum_{j=0}^{k-2}\left(\begin{array}{l}
k \\
j
\end{array}\right) \frac{n^{j} \alpha^{k-j}}{(n+\beta)^{k}} M_{n}\left(e_{j} ; z\right)+\frac{k n^{k-1} \alpha}{(n+\beta)^{k}}\left[M_{n}\left(e_{k-1} ; z\right)-e_{k-1}(z)\right] \\
& +\frac{k n^{k-1} \alpha}{(n+\beta)^{k}} z^{k-1}-\sum_{j=0}^{k-2}\left(\begin{array}{c}
k \\
j
\end{array}\right) \frac{n^{j} \beta^{k-j}}{(n+\beta)^{k}} M_{n}\left(e_{k} ; z\right) \\
& -\frac{k n^{k-1} \beta}{(n+\beta)^{k}}\left[M_{n}\left(e_{k} ; z\right)-e_{k}(z)\right]-\frac{k n^{k-1} \beta}{(n+\beta)^{k}} z^{k}-\frac{\alpha-\beta z}{n} k z^{k-1} \\
= & \sum_{j=0}^{k-2}\left(\begin{array}{l}
k \\
j
\end{array}\right) \frac{n^{j} \alpha^{k-j}}{(n+\beta)^{k}} M_{n}\left(e_{j} ; z\right)+\frac{k n^{k-1} \alpha}{(n+\beta)^{k}}\left[M_{n}\left(e_{k-1} ; z\right)-e_{k-1}(z)\right] \\
& -\sum_{j=0}^{k-2}\left(\begin{array}{l}
k \\
j
\end{array}\right) \frac{n^{j} \beta^{k-j}}{(n+\beta)^{k}} M_{n}\left(e_{k} ; z\right)-\frac{k n^{k-1} \beta}{(n+\beta)^{k}}\left[M_{n}\left(e_{k} ; z\right)-e_{k}(z)\right] \\
& -\left[\frac{1}{n}-\frac{n^{k-1}}{(n+\beta)^{k}}\right] k \alpha z^{k-1}+\left[\frac{1}{n}-\frac{n^{k-1}}{(n+\beta)^{k}}\right] k \beta z^{k} .
\end{aligned}
$$

By the proof of [11, Corollary 3], for any $k \in \mathbf{N},|z| \leq r, r \geq 1$, we have

$$
\left|M_{n}\left(e_{k} ; z\right)\right| \leq r^{k}, \quad\left|M_{n}\left(e_{k} ; z\right)-e_{k}\right| \leq \frac{2 k^{2}}{n} r^{k}
$$

Hence, for any $k \geq 2,|z| \leq r, r \geq 1$, we can get

$$
\begin{aligned}
& \left|\sum_{j=0}^{k-2}\left(\begin{array}{c}
k \\
j
\end{array}\right) \frac{n^{j} \alpha^{k-j}}{(n+\beta)^{k}} M_{n}\left(e_{j} ; z\right)\right| \\
& \quad \leq \sum_{j=0}^{k-2}\left(\begin{array}{l}
k \\
j
\end{array}\right) \frac{n^{j} \alpha^{k-j}}{(n+\beta)^{k}} r^{k-2} \\
& \quad=\sum_{j=0}^{k-2} \frac{k(k-1)}{(k-j)(k-j-1)}\left(\begin{array}{c}
k-2 \\
j
\end{array}\right) \frac{n^{j} \alpha^{k-2-j}}{(n+\beta)^{k-2}} \cdot \frac{\alpha^{2}}{(n+\beta)^{2}} r^{k-2} \\
& \quad \leq \frac{k(k-1)}{2} \cdot \frac{\alpha^{2}}{(n+\beta)^{2}} \sum_{j=0}^{k-2}\left(\begin{array}{c}
k-2 \\
j
\end{array}\right) \frac{n^{j} \alpha^{k-2-j}}{(n+\beta)^{k-2}} r^{k-2} \\
& \quad \leq \frac{k(k-1)}{2} \cdot \frac{\alpha^{2}}{(n+\beta)^{2}} r^{k-2}
\end{aligned}
$$

and

$$
\left|\frac{k n^{k-1} \alpha}{(n+\beta)^{k}}\left[M_{n}\left(e_{k-1} ; z\right)-e_{k-1}(z)\right]\right| \leq \frac{2 k(k-1)^{2} \alpha}{n(n+\beta)} r^{k-1} .
$$

Also, using

$$
\frac{1}{n}-\frac{n^{k-1}}{(n+\beta)^{k}}=\frac{\sum_{j=0}^{k-1}\left(\begin{array}{l}
k \\
j
\end{array}\right) n^{j} \beta^{k-j}}{n(n+\beta)^{k}} \leq \frac{k \beta}{n(n+\beta)}
$$


for any $k \geq 2,|z| \leq r, r \geq 1$, we get

$$
\begin{aligned}
& \left|M_{n}^{(\alpha, \beta)}\left(e_{k} ; z\right)-M_{n}\left(e_{k} ; z\right)-\frac{\alpha-\beta z}{n} k z^{k-1}\right| \\
& \leq \frac{k(k-1)}{2} \cdot \frac{\alpha^{2}}{(n+\beta)^{2}} r^{k-2}+\frac{2 k(k-1)^{2} \alpha}{n(n+\beta)} r^{k-1}+\frac{k(k-1)}{2} \cdot \frac{\beta^{2}}{(n+\beta)^{2}} r^{k} \\
& \quad+\frac{2 k^{3} \beta}{n(n+\beta)} r^{k}+\frac{k^{2} \alpha \beta}{n(n+\beta)} r^{k-1}+\frac{k^{2} \beta^{2}}{n(n+\beta)} r^{k} \\
& =\frac{r^{k-1}}{n(n+\beta)}\left[2 k(k-1)^{2} \alpha+2 k^{3} \beta r+k^{2} \alpha \beta+k^{2} \beta^{2} r\right] \\
& \quad+\frac{r^{k-2}}{(n+\beta)^{2}} \cdot \frac{k(k-1)\left(\alpha^{2}+\beta^{2} r^{2}\right)}{2} .
\end{aligned}
$$

On the other hand, when $k=1$, using Lemma 1 and $M_{n}\left(e_{1} ; z\right)=\frac{n z}{n+1}$ (see [19]), by a simple calculation, we can get $\left|M_{n}^{(\alpha, \beta)}\left(e_{1} ; z\right)-M_{n}\left(e_{1} ; z\right)-\frac{\alpha-\beta z}{n}\right| \leq \frac{1}{n(n+\beta)}\left(2 \beta r+\alpha \beta+\beta^{2} r\right)$.

So, for any $k \in \mathbf{N},|z| \leq r, r \geq 1$, we have

$$
\begin{gathered}
\left|M_{n}^{(\alpha, \beta)}\left(e_{k} ; z\right)-M_{n}\left(e_{k} ; z\right)-\frac{\alpha-\beta z}{n} k z^{k-1}\right| \\
\leq \frac{r^{k-1}}{n(n+\beta)}\left[2 k(k-1)^{2} \alpha+2 k^{3} \beta r+k^{2} \alpha \beta+k^{2} \beta^{2} r\right] \\
\quad+\frac{r^{k-2}}{(n+\beta)^{2}} \cdot \frac{k(k-1)\left(\alpha^{2}+\beta^{2} r^{2}\right)}{2} .
\end{gathered}
$$

Hence, we have

$$
\left|I_{2}\right| \leq \frac{M_{r, 1}^{(\alpha, \beta)}(f)}{n(n+\beta)}+\frac{M_{r, 2}^{(\alpha, \beta)}(f)}{(n+\beta)^{2}},
$$

where $M_{r, 1}^{(\alpha, \beta)}(f)=\sum_{k=1}^{\infty}\left|c_{k}\right|\left[2 k(k-1)^{2} \alpha+2 k^{3} \beta r+k^{2} \alpha \beta+k^{2} \beta^{2} r\right] r^{k-1}, M_{r, 2}^{(\alpha, \beta)}(f)=$ $\sum_{k=1}^{\infty}\left|c_{k}\right| \frac{k(k-1)\left(\alpha^{2}+\beta^{2} r^{2}\right)}{2} r^{k-2}$.

In conclusion, we obtain

$$
\begin{aligned}
& \left|M_{n}^{(\alpha, \beta)}(f ; z)-f(z)-\frac{\alpha-(1+\beta) z}{n} f^{\prime}(z)-\frac{z(1-z)}{n} f^{\prime \prime}(z)\right| \\
& \quad \leq\left|I_{1}\right|+\left|I_{2}\right| \leq \frac{M_{r}(f)}{n^{2}}+\frac{M_{r, 1}^{(\alpha, \beta)}(f)}{n(n+\beta)}+\frac{M_{r, 2}^{(\alpha, \beta)}(f)}{(n+\beta)^{2}} .
\end{aligned}
$$

In the following theorem, we obtain the exact order of approximation.

Theorem 3 Let $0 \leq \alpha \leq \beta, R>1, D_{R}=\{z \in \mathbf{C}:|z|<R\}$. Suppose that $f: D_{R} \rightarrow \mathbf{C}$ is analytic in $D_{R}$. Iff is not a polynomial of degree 0 , then for any $r \in[1, R)$, we have

$$
\left\|M_{n}^{(\alpha, \beta)}(f ; \cdot)-f\right\|_{r} \geq \frac{C_{r}^{(\alpha, \beta)}(f)}{n}, \quad n \in \mathbf{N}
$$

where $\|f\|_{r}=\max \{|f(z)| ;|z| \leq r\}$ and the constant $C_{r}^{(\alpha, \beta)}(f)>0$ depends on $f, r$ and $\alpha, \beta$, but it is independent of $n$. 
Proof Define $e_{1}(z)=z$ and

$$
H_{n}^{(\alpha, \beta)}(f ; z)=M_{n}^{(\alpha, \beta)}(f ; z)-f(z)-\frac{\alpha-(1+\beta) z}{n} f^{\prime}(z)-\frac{z(1-z)}{n} f^{\prime \prime}(z) .
$$

For all $z \in D_{R}$ and $n \in \mathbf{N}$, we have

$$
\begin{aligned}
& M_{n}^{(\alpha, \beta)}(f ; z)-f(z) \\
& \quad=\frac{1}{n}\left\{[\alpha-(1+\beta) z] f^{\prime}(z)+z(1-z) f^{\prime \prime}(z)+\frac{1}{n}\left[n^{2} H_{n}^{(\alpha, \beta)}(f ; z)\right]\right\} .
\end{aligned}
$$

In view of the property $\|F+G\|_{r} \geq\left|\|F\|_{r}-\|G\|_{r}\right| \geq\|F\|_{r}-\|G\|_{r}$, it follows

$$
\begin{aligned}
& \left\|M_{n}^{(\alpha, \beta)}(f ; \cdot)-f\right\|_{r} \\
& \quad \geq \frac{1}{n}\left\{\left\|\left[\alpha-(1+\beta) e_{1}\right] f^{\prime}+e_{1}\left(1-e_{1}\right) f^{\prime \prime}\right\|_{r}-\frac{1}{n}\left[n^{2}\left\|H_{n}^{(\alpha, \beta)}(f ; \cdot)\right\|_{r}\right]\right\} .
\end{aligned}
$$

Considering the hypothesis that $f$ is not a polynomial of degree 0 in $D_{R}$, we have

$$
\left\|\left[\alpha-(1+\beta) e_{1}\right] f^{\prime}+e_{1}\left(1-e_{1}\right) f^{\prime \prime}\right\|_{r}>0 .
$$

Indeed, supposing the contrary, it follows that

$$
[\alpha-(1+\beta) z] f^{\prime}(z)+z(1-z) f^{\prime \prime}(z)=0 \quad \text { for all } z \in \bar{D}_{r} .
$$

Defining $y(z)=f^{\prime}(z)$ and looking for the analytic function $y(z)$ under the form $y(z)=$ $\sum_{k=0}^{\infty} a_{k} z^{k}$, after replacement in the differential equation, the coefficients identification method immediately leads to $a_{k}=0$ for all $k \in \mathbf{N} \cup\{0\}$. This implies that $y(z)=0$ for all $z \in \bar{D}_{r}$ and therefore $f$ is constant on $\bar{D}_{r}$, a contradiction with the hypothesis.

Using inequality (4), we get

$$
n^{2}\left\|H_{n}^{(\alpha, \beta)}(f ; \cdot)\right\|_{r} \leq N_{r}^{(\alpha, \beta)}(f),
$$

where $N_{r}^{(\alpha, \beta)}(f)=M_{r}(f)+M_{r, 1}^{(\alpha, \beta)}(f)+M_{r, 2}^{(\alpha, \beta)}(f)$.

Therefore, there exists an index $n_{0}$, depending only on $f, r$ and $\alpha, \beta$, such that for all $n \geq n_{0}$, we have

$$
\begin{aligned}
& \left\|\left[\alpha-(1+\beta) e_{1}\right] f^{\prime}+e_{1}\left(1-e_{1}\right) f^{\prime \prime}\right\|_{r}-\frac{1}{n}\left[n^{2}\left\|H_{n}^{(\alpha, \beta)}(f ; \cdot)\right\|_{r}\right] \\
& \quad \geq \frac{1}{2}\left\|\left[(1+\alpha)-(2+\beta) e_{1}\right] f^{\prime}+e_{1}\left(1-e_{1}\right) f^{\prime \prime}\right\|_{r}
\end{aligned}
$$

which implies

$$
\left\|M_{n}^{(\alpha, \beta)}(f ; \cdot)-f\right\|_{r} \geq \frac{1}{2 n}\left\|\left[\alpha-(1+\beta) e_{1}\right] f^{\prime}+e_{1}\left(1-e_{1}\right) f^{\prime \prime}\right\|_{r} \quad \text { for all } n \geq n_{0} .
$$


For $n \in\left\{1,2, \ldots, n_{0}-1\right\}$, we have

$$
\left\|M_{n}^{(\alpha, \beta)}(f ; \cdot)-f\right\|_{r} \geq \frac{W_{r, n}^{(\alpha, \beta)}(f)}{n}
$$

where $W_{r, n}^{(\alpha, \beta)}(f)=n\left\|M_{n}^{(\alpha, \beta)}(f ; \cdot)-f\right\|_{r}>0$.

As a conclusion, we have

$$
\left\|M_{n}^{(\alpha, \beta)}(f ; \cdot)-f\right\|_{r} \geq \frac{C_{r}^{(\alpha, \beta)}(f)}{n} \quad \text { for all } n \in \mathbf{N}
$$

where

$$
\begin{aligned}
C_{r}^{(\alpha, \beta)}(f)= & \min \left\{W_{r, 1}^{(\alpha, \beta)}(f), W_{r, 2}^{(\alpha, \beta)}(f), \ldots, W_{r, n_{0}-1}^{(\alpha, \beta)}(f),\right. \\
& \left.\frac{1}{2}\left\|\left[\alpha-(1+\beta) e_{1}\right] f^{\prime}+e_{1}\left(1-e_{1}\right) f^{\prime \prime}\right\|_{r}\right\},
\end{aligned}
$$

this completes the proof.

Combining Theorem 3 with Theorem 1, we get the following result.

Corollary 1 Let $0 \leq \alpha \leq \beta, R>1, D_{R}=\{z \in \mathbf{C}:|z|<R\}$. Suppose that $f: D_{R} \rightarrow \mathbf{C}$ is analytic in $D_{R}$. Iff is not a polynomial of degree 0 , then for any $r \in[1, R)$, we have

$$
\left\|M_{n}^{(\alpha, \beta)}(f ; \cdot)-f\right\|_{r} \asymp \frac{1}{n}, \quad n \in \mathbf{N},
$$

where $\|f\|_{r}=\max \{|f(z)| ;|z| \leq r\}$ and the constants in the equivalence depend on $f, r$ and $\alpha$, $\beta$, but they are independent of $n$.

Theorem 4 Let $0 \leq \alpha \leq \beta, R>1, D_{R}=\{z \in \mathbf{C}:|z|<R\}$. Suppose that $: D_{R} \rightarrow \mathbf{C}$ is analytic in $D_{R}$. Also, let $1 \leq r<r_{1}<R$ and $p \in \mathbf{N}$ be fixed. Iff is not a polynomial of degree $\leq p-1$, then we have

$$
\left\|\left(M_{n}^{(\alpha, \beta)}(f ; \cdot)\right)^{(p)}-f^{(p)}\right\|_{r} \asymp \frac{1}{n}, \quad n \in \mathbf{N},
$$

where $\|f\|_{r}=\max \{|f(z)| ;|z| \leq r\}$ and the constants in the equivalence depend on $f, r, r_{1}, p$, $\alpha$ and $\beta$, but they are independent of $n$.

Proof Taking into account the upper estimate in Theorem 1, it remains to prove the lower estimate only. Denoting by $\Gamma$ the circle of radius $r_{1}>r$ and center 0 , by Cauchy's formula, it follows that for all $|z| \leq r$ and $n \in \mathbf{N}$, we have

$$
\left(M_{n}^{(\alpha, \beta)}(f ; z)\right)^{(p)}-f^{(p)}(z)=\frac{p !}{2 \pi i} \int_{\Gamma} \frac{M_{n}^{(\alpha, \beta)}(f ; v)-f(v)}{(v-z)^{p+1}} d v .
$$


Keeping the notation there for $H_{n}^{(\alpha, \beta)}(f ; z)$, for all $n \in \mathbf{N}$, we have

$$
\begin{aligned}
& M_{n}^{(\alpha, \beta)}(f ; z)-f(z) \\
& \quad=\frac{1}{n}\left\{[\alpha-(1+\beta) z] f^{\prime}(z)+z(1-z) f^{\prime \prime}(z)+\frac{1}{n}\left[n^{2} H_{n}^{(\alpha, \beta)}(f ; z)\right]\right\} .
\end{aligned}
$$

By using Cauchy's formula, for all $v \in \Gamma$, we get

$$
\begin{aligned}
\left(M_{n}^{(\alpha, \beta)}(f ; z)\right)^{(p)}-f^{(p)}(z)= & \frac{1}{n}\left\{\left[(\alpha-(1+\beta) z) f^{\prime}(z)+z(1-z) f^{\prime \prime}(z)\right]^{(p)}\right. \\
& \left.+\frac{1}{n} \cdot \frac{p !}{2 \pi i} \int_{\Gamma} \frac{n^{2} H_{n}^{(\alpha, \beta)}(f ; v)}{(v-z)^{p+1}} d v\right\}
\end{aligned}
$$

Passing now to $\|\cdot\|_{r}$ and denoting $e_{1}(z)=z$, it follows

$$
\begin{aligned}
\left\|\left(M_{n}^{(\alpha, \beta)}(f ; \cdot)\right)^{(p)}-f^{(p)}\right\|_{r} \geq & \frac{1}{n}\left[\left\|\left[\left(\alpha-(1+\beta) e_{1}\right) f^{\prime}+e_{1}\left(1-e_{1}\right) f^{\prime \prime}\right]^{(p)}\right\|_{r}\right. \\
& \left.-\frac{1}{n}\left\|\frac{p !}{2 \pi i} \int_{\Gamma} \frac{n^{2} H_{n}^{(\alpha, \beta)}(f ; v)}{(v-\cdot)^{p+1}} d v\right\|_{r}\right] .
\end{aligned}
$$

Since for any $|z| \leq r$ and $v \in \Gamma$ we have $|v-z| \geq r_{1}-r$, so, by inequality (5), we get

$$
\begin{aligned}
\left\|\frac{p !}{2 \pi i} \int_{\Gamma} \frac{n^{2} H_{n}^{(\alpha, \beta)}(f ; v)}{(v-\cdot)^{p+1}} d v\right\|_{r} & \leq \frac{p !}{2 \pi} \cdot \frac{2 \pi r_{1} n^{2}\left\|H_{n}^{(\alpha, \beta)}(f ; \cdot)\right\|_{r_{1}}}{\left(r_{1}-r\right)^{p+1}} \\
& \leq \frac{N_{r_{1}}^{(\alpha, \beta)}(f) p ! r_{1}}{\left(r_{1}-r\right)^{p+1}}
\end{aligned}
$$

where $N_{r_{1}}^{(\alpha, \beta)}(f)=M_{r_{1}}(f)+M_{r_{1}, 1}^{(\alpha, \beta)}(f)+M_{r_{1}, 2}^{(\alpha, \beta)}(f)$.

Taking into account that the function $f$ is analytic in $D_{R}$, by following exactly the lines in Gal [5], seeing also the book Gal [6, pp.77-78] (where it is proved that $\|\left[\left(\alpha-\beta e_{1}\right) f^{\prime}+\right.$ $\left.\left.\frac{e_{1}\left(1-e_{1}\right)}{2} f^{\prime \prime}\right]^{(p)} \|_{r}>0\right)$, we have

$$
\left\|\left[\left(\alpha-(1+\beta) e_{1}\right) f^{\prime}+e_{1}\left(1-e_{1}\right) f^{\prime \prime}\right]^{(p)}\right\|_{r}>0
$$

In continuation, reasoning exactly as in the proof of Theorem 3, we can get the desired conclusion.

\section{Competing interests}

The authors declare that they have no competing interests.

\section{Authors' contributions}

All authors contributed equally and significantly in writing this article. All authors read and approved the final manuscript.

\section{Author details}

'Department of Mathematics and Computer Science, Wuyi University, Wuyishan, 354300, China. ${ }^{2}$ Department of Mathematics, Xiamen University, Xiamen, 361005, China. 


\section{Acknowledgements}

The authors are most grateful to the editor and anonymous referee for careful reading of the manuscript and valuable suggestions which helped in improving an earlier version of this paper. This work is supported by the National Natural Science Foundation of China (Grant no. 61170324), the Class A Science and Technology Project of Education Department of Fujian Province of China (Grant no. JA12324), and the Natural Science Foundation of Fujian Province of China (Grant no. 2013J01017)

Received: 29 April 2013 Accepted: 23 August 2013 Published: 23 September 2013

\section{References}

1. Lorentz, GG: Bernstein Polynomials, 2nd edn. Chelsea, New York (1986)

2. Gal, SG: Voronovskaja's theorem and iterations for complex Bernstein polynomials in compact disks. Mediterr. J. Math. 5, 253-272 (2008)

3. Anastassiou, GA, Gal, SG: Approximation by complex Bernstein-Schurer and Kantorovich-Schurer polynomials in compact disks. Comput. Math. Appl. 58, 734-743 (2009)

4. Gal, SG: Approximation by complex Bernstein-Kantorovich and Stancu-Kantorovich polynomials and their iterates in compact disks. Rev. Anal. Numér. Théor. Approx. 37, 159-168 (2008)

5. Gal, SG: Exact orders in simultaneous approximation by complex Bernstein-Stancu polynomials. Rev. Anal. Numér Théor. Approx. 37, 47-52 (2008)

6. Gal, SG: Approximation by Complex Bernstein and Convolution Type Operators. World Scientific, Singapore (2009)

7. Gal, SG: Exact orders in simultaneous approximation by complex Bernstein polynomials. J. Concr. Appl. Math. 7, 215-220 (2009)

8. Gal, SG: Approximation by complex Bernstein-Stancu polynomials in compact disks. Results Math. 53, 245-256 (2009)

9. Gal, SG: Approximation by complex genuine Durrmeyer type polynomials in compact disks. Appl. Math. Comput. 217, 1913-1920(2010)

10. Gal, SG: Approximation by complex Bernstein-Durrmeyer polynomials with Jacobi weights in compact disks. Math Balk. 24, 103-110 (2010)

11. Gal, SG, Gupta, V: Approximation by a Durrmeyer-type operator in compact disks. Ann. Univ. Ferrara 57, 261-274 (2011)

12. Gal, SG, Gupta, V: Approximation by complex beta operators of first kind in strips of compact disks. Mediterr. J. Math. 10, 31-39(2013)

13. Gupta, V: Approximation properties by Bernstein-Durrmeyer type operators. Complex Anal. Oper. Theory 7, 363-374 (2013)

14. Gal, SG, Gupta, V, et al.: Approximation by complex Baskakov-Stancu operators in compact disks. Rend. Circ. Mat. Palermo 61, 153-165 (2012)

15. Gal, SG, Mahmudov, NI, Kara, M: Approximation by complex $q$-Szász-Kantorovich operators in compact disks, $q>1$. Complex Anal. Oper. Theory (2012). doi:10.1007/s11785-012-0257-3

16. Mahmudov, NI: Approximation properties of complex $q$-Szász-Mirakjan operators in compact disks. Comput. Math Appl. 60, 1784-1791 (2010)

17. Mahmudov, NI, Gupta, V: Approximation by genuine Durrmeyer-Stancu polynomials in compact disks. Math Comput. Model. 55, 278-285 (2012)

18. Ren, MY, Zeng, XM: Approximation by a kind of complex modified $q$-Durrmeyer type operators in compact disks. J. Inequal. Appl. 2012, 212 (2012). doi:10.1186/1029-242X-2012-212

19. Gupta, V, Maheshwari, P: Bezier variant of a new Durrmeyer type operators. Riv. Mat. Univ. Parma 7, 9-21 (2003)

doi:10.1186/1029-242X-2013-442

Cite this article as: Ren et al.: Approximation by complex Durrmeyer-Stancu type operators in compact disks. Journal of Inequalities and Applications 2013 2013:442.

\section{Submit your manuscript to a SpringerOpen ${ }^{\circ}$ journal and benefit from:}

- Convenient online submission

Rigorous peer review

- Immediate publication on acceptance

Open access: articles freely available online

- High visibility within the field

- Retaining the copyright to your article 